\title{
A pattern matching technique for measuring sediment displacement levels
}

\author{
by R. J. Munro ${ }^{1}$, S. B. Dalziel ${ }^{1} \&$ H. Jehan ${ }^{2}$. \\ ${ }^{1}$ Department of Applied Mathematics and Theoretical Physics, University of Cam- \\ bridge, Wilberforce Road, Cambridge CB3 0WA, UK. \\ ${ }^{2}$ ENSTA, Départment UME, 32 Boulevard Victor, 75015 Paris, France.
}

\begin{abstract}
This paper describes a novel technique for obtaining accurate, high (spatial) resolution measurements of sediment redeposition levels. A sequence of different random patterns are projected onto sediment layer and captured using a high resolution camera, producing a set of reference images. The same patterns are used to obtain a corresponding sequence of deformed images after a region of the sediment layer has been displaced and redeposited, allowing the use of a high accuracy pattern matching algorithm to quantify the distribution of redeposited sediment. A set of experiments, using the impact of a vortex ring with a glass ballotini particle layer as the resuspension mechanism, are described to test and illustrate the technique. The accuracy of the procedure is assessed using a known crater profile, manufactured to simulate the features of the craters observed in the experiments.
\end{abstract}

\section{Introduction}

The development of image processing hardware and associated software tools has led to a revolution in the scope and quantity of quantitative information that may be gathered from laboratory experiments. While progress has been significant over the last decade, most effort has been focused on obtaining velocity fields, even though the underlying techniques and technologies have a much broader application.

In this paper we explore the use of pattern matching techniques to provide accurate, quantitative, non-intrusive measurements of changes in the depth of a layer of sediment due to a resuspension event. The key focus here is on the measurement technique itself, rather than the dynamics of the resuspension event that motivated the development of the technique.

In recent years, pattern matching techniques have been employed in most if not all branches of science. The fundamental idea is to obtain, or at least assess, the correspondence between two sets of data. The aim may be to seek a simple binary yes/no answer, or to determine the set of independent parameters that yields the best match between the two data sets. 
In fluid dynamics, the most common use of pattern matching techniques is in so-called particle image velocimetry (PIV), where the aim is to determine the vector displacement field that gives the best correspondence between two images. In this case each image contains a pattern, typically created by the illumination of randomly distributed, neutrally buoyant particles suspended in the fluid, that is advected and distorted by the fluid flow. Consequently, the pattern displacements are directly related to the velocity field of the fluid. The problem is complicated in most flows by particles entering or leaving the illuminated volume between successive frames, leading to an increased probability of spurious velocities being returned.

A substantial volume of literature has grown up around PIV, and includes many variations on the same basic technique, often with reinvention of the same ideas by different authors. An early review of the subject is provided by Adrian (1991), with more recent contributions by Raffel et al. (1998) and a review article by Cowen \& Sveen (2004). Despite their potential value, pattern matching techniques have not found wide use for other measurements in experimental fluid dynamics. The work by Dalziel et al. (2000), who utilized pattern matching techniques to measure the density perturbations induced by internal gravity waves is one of only a few such applications.

The aim in this paper is to provide a simple yet accurate technique for providing high-resolution measurements of the deformation of a particle bed due to the suspension and subsequent redeposition of particles caused by the impact of a vortex ring with a sediment bed. The measurement technique described here, however, has the potential for being applied to a wide scope of problems.

A number of other techniques are available for measuring the thickness of a layer of sediment in the laboratory. The majority of researchers have employed either acoustic or laser range finding devices (e.g. Williams \& Jackson (1998) and Irish \& White (1998)). However, such techniques are unpractical when a high degree of spatial resolution is required over a relatively large area of the sediment layer. High resolution measurements, both temporal and spatial, have been made electrically (e.g. Rooij et al. (1999); Munro \& Dalziel (2004a)) using an array of electrodes in the base of the tank below the sediment layer. With a reference electrode placed in the water above the layer, the resistance of the particles above each of the base electrodes can then be measured, and used to calculate the sediment depth at that point. The problem has also been approached using an optical technique by Munro \& Dalziel (2004b), who considered the optical thickness of the sediment layer in a setup very similar to the one described here. One limitation of the latter two methods is that for deep layers the signal (i.e. either voltage or intensity) becomes saturated, hence limiting the dynamic range of the measurements.

The basic experimental apparatus are introduced in section 2, followed 
by the details of the pattern matching algorithm and calibration procedure in section 3. Results of a validation study are reported in section 4, and a brief exposition of some of the results from the resuspension experiments are given in section 5. The paper concludes with the discussion in section 6 .

\section{Apparatus}

The basic concept behind the pattern matching technique described here is to use a randomly structured pattern projected vertically down onto the surface of a sediment layer. When viewed at an angle oblique to that of the projection angle, any localized redistribution of sediment will result in a displacement in the corresponding region of the image of the projected pattern. The magnitude and direction of the displacement at any given point in the pattern is directly related to the change in depth of the sediment at that point. Hence, a reference image taken of the pattern projected onto the undisturbed sediment layer, and a distorted image taken after the sediment has been redeposited, allow highly accurate pattern matching techniques (such as those used in synthetic schlieren (Dalziel et al., 2000)) to provide accurate measurements of the corresponding displacement field at each pixel location in the image. A simple but accurate calibration can then convert these image displacements into the corresponding changes in sediment depth.

The level of spatial resolution that can be achieved in the pattern matching procedure scales with the degree of spatial texture in the random patterns used, together with the spatial resolution of the camera. That is, patterns with a fine-scale structure are more likely to detect distortion over smaller scales than patterns with a more coarse structure. For the purpose of the present study it was found that accurate results were obtained using a random pattern ${ }^{1}$ of white dots on a black background; typical examples of the patterns used are shown in figure 1 , together with an image of the fine-scale pattern projected onto the sediment bed.

Figure 2 shows the basic experimental setup. A small Perspex tank, with dimensions $30 \mathrm{~cm} \times 30 \mathrm{~cm} \times 40 \mathrm{~cm}$, was filled with tap water to a level $7 \mathrm{~cm}$ below the top of the tank. Small glass ballotini, with diameter range $0.02-0.03 \mathrm{~cm}$ and density $\rho=2.4 \mathrm{~g} \mathrm{~cm}^{-3}$, were added and formed into a smooth, even layer of sediment $1 \mathrm{~cm}$ thick.

The accuracy of the measured displacement fields is limited by the optics of forming the pattern on the particle bed, the resolution of the imaging hardware utilized, and by the pattern matching algorithm. As shall be discussed in section 3 , thermal noise in the laboratory can also affect the accuracy of the results.

\footnotetext{
${ }^{1}$ The use of a random pattern eliminates the problem of aliasing which may occur when using a pattern with a regular or periodic structure.
} 

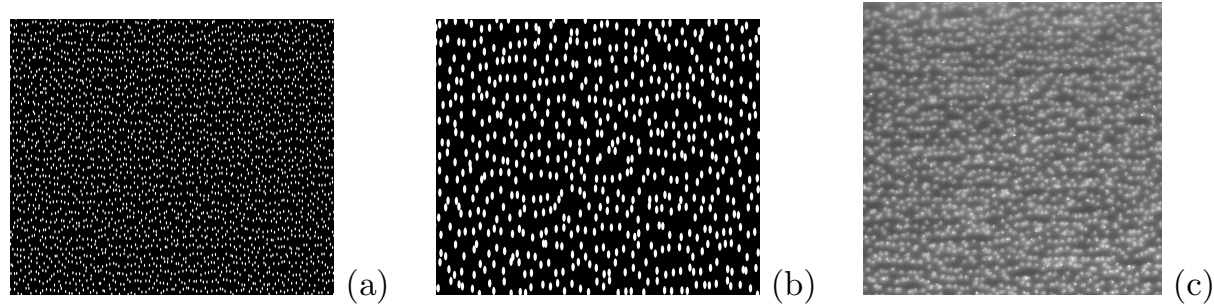

Figure 1: Images showing the typical random patterns used for the pattern matching procedure: (a) fine-scale texture, (b) coarse-scale texture, (c) the fine-scale image projected onto the undisturbed sediment layer.

While it is not necessary in pattern matching for the image of the projected pattern to be sharp, there is an underlying assumption that it forms on a surface that is smooth and continuous at length scales that can be seen by the imaging hardware, and that the optical properties of the surface where the pattern forms do not change due to the resuspension or sedimentation. For this present study we were using transparent glass ballotini with a refractive index of $n \approx 1.5$. As such, the incident light of the projected pattern was not scattered directly back to the video camera from a diffuse reflection, but rather experienced multiple refraction and reflection events, penetrating a number of particle diameters below the surface, before the light reached the video camera. A direct consequence of this is that it is not possible to form a sharp image on the surface of the particle bed (see figure 1(c)). Moreover, the image ultimately formed depends on the precise configuration of particles close to the surface. As this configuration changes due to a redistribution of particles, the image formed on the bed will suffer a distortion that does not depend simply on the elevation of the bed.

This inability to form a consistent image at the surface of the bed is of little importance if the size of the particles is small compared with the resolution of the captured images and the bed displacements. However, in the present study we aim to detect changes in bed thickness of a similar order to the particle diameter, and so must take measures to reduce the influence of this effect. Moreover, as will be seen below, each pixel in the video camera images corresponds to approximately the dimensions of a single particle on the sediment bed. Hence, the image formed is influenced by individual particles.

The approach adopted here is to utilize a sequence of different random patterns projected onto the particle bed. Each pattern will interact with the particle configuration where it is formed in a different manner, and the distortion 


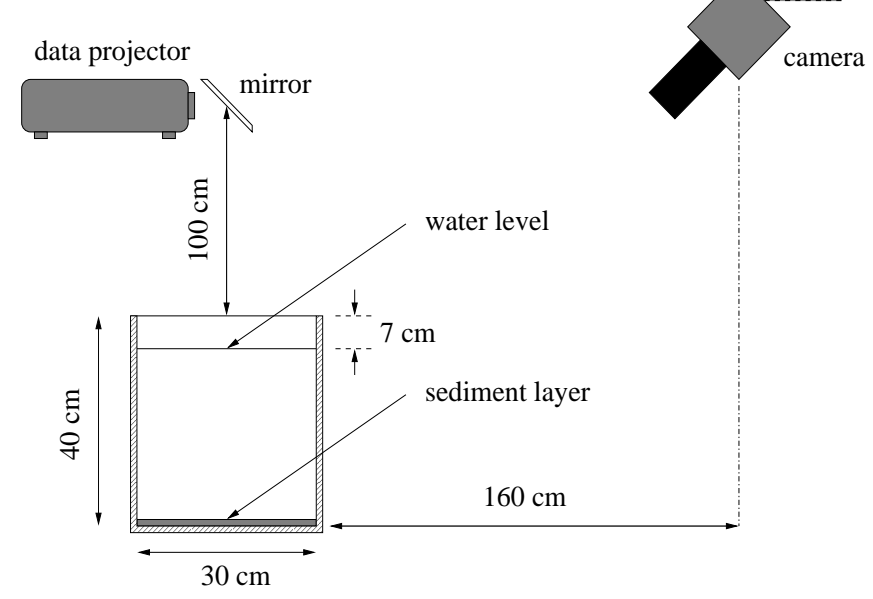

Figure 2: Setup of apparatus for pattern matching procedure.

of the pattern induced by any change in particle configuration will be different for each image. We then use an ensemble average of the measurements obtained from these different patterns to reduce the effect of the particle configuration on our measurements.

The use of multiple random patterns has an additional advantage in reducing errors in the pattern matching algorithm, particularly the errors associated with measuring extremely small displacements (see section 3 for further details). For this present work, a total of eleven patterns were constructed, nine with a fine-scale structure such as the pattern shown in figure 1(a), and two coarse-scale patterns, one of which is shown in figure 1(b). The final results were obtained by processing each image separately, then combining the estimated displacement fields.

Because of the need to project the sequence of patterns twice - once before the resuspension event, and once after - with guaranteed alignment between them, we elected to use a standard data projector (with $1024 \times 768$ pixel resolution) connected to a PC. The projector was oriented horizontally and located approximately $140 \mathrm{~cm}$ above the sediment layer, close to the minimum focal distance for the projector's lens. A small front-silvered mirror was placed in front of the projector to redirect the pattern vertically downwards; this eliminated the need for complicated brackets to hold the projector in a vertical position. The room was darkened to optimize the contrast in the images.

The pattern was generated using a simple PostScript procedure, giving a 
random perturbation to a regular array, and rendered using GhostView. The aspect ratio of the pattern was set so that the individual dots were elliptical, hence appearing to be circular when viewed from the oblique angle of the camera (see figure 1(a) and (b)). The individual features in the image were elliptical in the PostScript, but these became pixelized at the resolution of the data projector.

In a limited number of cases the projector did not align the patterns in the reference and corresponding distorted images; typically there was a small 5-10 pixel horizontal shift. This was remedied by including a vertical white stripe in each of the patterns used. The stripe was positioned to appear along the extreme right hand edge of the captured images, corresponding to a region of the bed which would remain undisturbed. The stripe was easily identified in each of the reference and distorted images, and used to eliminate any shift in the patterns. In the captured images, the stripe was approximately 40 pixels wide.

The images were captured using a digital Camelia 8 Megapixel camera with $3500 \times 2300$ pixel resolution at 12 bits per pixel, used in conjunction with a Nikon Zoom-Nikkor $35 \sim 135 \mathrm{~mm}$ F3.5 4.5 lens and an LCD shutter (the full frame sensor in the Camelia camera lacks the electronic shutter found in most CCD cameras). The camera was positioned on a tripod a distance of $160 \mathrm{~cm}$ from the front of the tank and was focused looking down onto the particle bed at an angle of approximately $45^{\circ}$. This angle was chosen so that the images produced were able to capture the deepest features of the craters, while the corresponding apparent displacements of the pattern obtained were sufficiently large to provide accurate measurements. It should be noted that, while the apparent displacement of the pattern will increase with shallower viewing angles, the spatial resolution will be diminished and the probability of regions being hidden by the sloping sides of the crater will increase. Due to the oblique view of the camera and the available lenses, we were unable to utilize the full vertical resolution of the camera, which was effectively halved. In the resulting images of the projected patterns a single dot diameter corresponds to about 10 pixels for the fine scale patterns (e.g. figure $1(\mathrm{c})$ ), that is each pixel images approximately $0.02 \mathrm{~cm} \times 0.03 \mathrm{~cm}$ pixels on the surface of the bed.

The vortex rings were generated using a circular tube of diameter $D=$ $3.9 \mathrm{~cm}$ connected to a bicycle track pump with a $2.8 \mathrm{~cm}$ bore (see figure 3 ). When the pump is actuated, air is forced into the circular tube, thus forcing a slug of water out of the open end. A boundary layer develops adjacent to the inner wall of the tube and quickly separates at the orifice causing vortical fluid to roll up and form the vortex ring; a comprehensive overview of experimental formation of vortex rings can be found in Glezer (1988) and Linden \& Turner (2001). The process was made repeatable by attaching the pump handle to a 


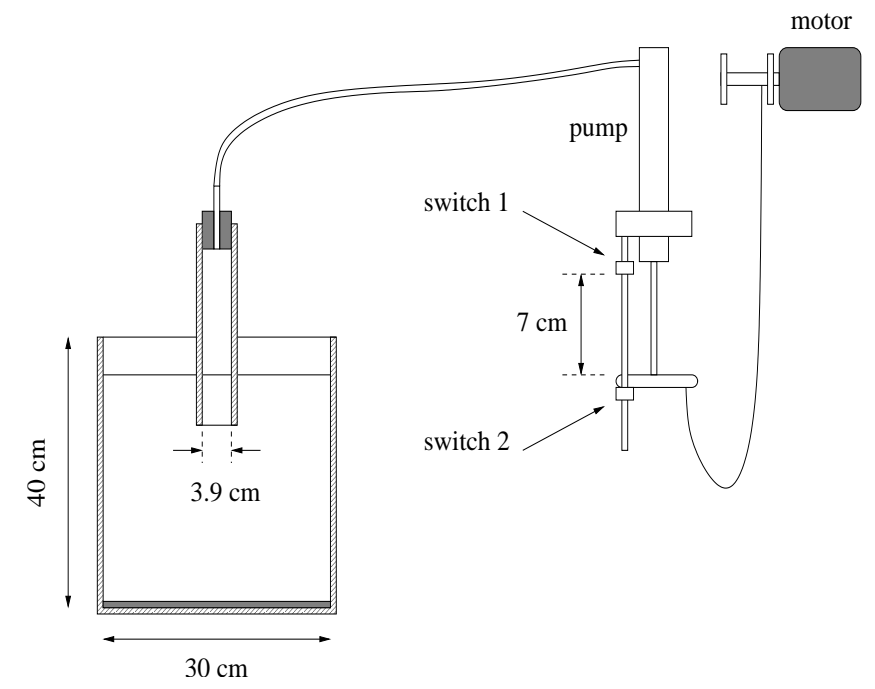

Figure 3: Schematic showing the setup for generating vortex rings.

length of cord which in turn was connected to a motor-driven spindle. When the motor is switched on the cord is wound around the spindle, actuating the pump handle. The speed of the motor was controlled by a manual switch with a wide range of settings. The length of the cord was set so that it did not begin to actuate the pump until the motor had time to reach a constant speed. The stroke length of the pump was held constant throughout the experiments at $7 \mathrm{~cm}$, which consistently produced a fluid slug with a length of $L \approx 4 \mathrm{~cm}$, i.e. $L / D \approx 1$. Note that for the limiting (inviscid) case of Hill's spherical vortex, $L / D=4$ (Linden \& Turner, 2001).

The system was calibrated so that the vortex ring Reynolds number, $R e=$ $D U / \nu$, could be determined by the stroke time $T[\mathrm{~s}]$ of the pump. (The stroke time was measured using an oscilloscope connected to two switches which could detect the start and end of the pump-handle stroke.) An extensive set of visualization experiments showed that once the vortex ring had propagated a distance of order $2 D$ from the orifice, the propagation speed was constant and remained so until the vortex ring was influenced by the bottom boundary a distance of $D$ from the tank floor. This constant propagation speed was adopted for the value of $U[\mathrm{~cm} / \mathrm{s}]$, and related to the stroke time by a simple power law.

The particle bed was prepared by carefully dragging a scraper across its surface. The scraper was maintained at a constant height above the floor of the tank by two $1 \mathrm{~cm}$ diameter stainless steel rods located along two opposing sides of the tank. This method produced a consistent mean thickness of the 

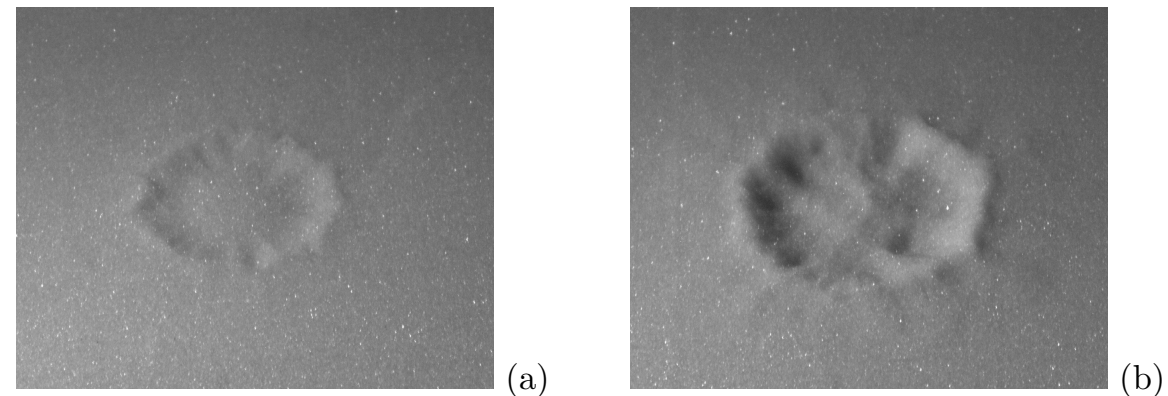

Figure 4: Images showing the redeposition crater produced by the impact of a vortex ring (a) $R e \approx 5000$, and (b) $R e \approx 28000$.

bed, although invariably the bed was not completely flat. We estimate the remaining features had a typical length scale of order of several centimetres in the plane of the bed, and depth of order comparable to the particle diameter (i.e. $0.02-0.03 \mathrm{~cm}$ ). Measurements of the redistribution of sediment were always made relative to this initial bed thickness. We believe that the small amplitude and long wavelength of the initial irregularities did not affect the dynamics of the vortex ring or the resuspension.

Once the reference images (one for each dot pattern) of the smoothed, horizontal particle layer had been taken, the tube was fixed vertically in the centre of the tank with the outlet a depth of $7 \mathrm{~cm}$ below the water surface. The motorized pump was set to a known stroke time and the motor activated. After the vortex ring had impacted the particle layer, and all resuspended material had been redeposited, the tube was removed so the images of the deformed particle bed could be taken ${ }^{2}$. This was repeated for a total of nine Reynolds numbers between $5000 \leqslant R e \leqslant 28000$. It is worth noting that, for the particles used here, no resuspension was produced by the impact for $R e \leqslant 5000$. Figure 4 shows two examples, at the lower and upper end of this range, of the redeposition crater produced by the vortex ring impact.

\section{Processing and calibration}

The pattern matching algorithm that was used to process the images is a further development of the one employed previously for synthetic schlieren by Dalziel et al. (2000). This algorithm is fundamentally similar to that used in

\footnotetext{
${ }^{2}$ Note that, since the images are projected vertically down into the tank, any discernible change in the free surface, or the water depth itself, will result in a distortion of the projected pattern on the sediment layer. Hence, before each image is captured care was taken to allow the free surface to become stationary, and that it was maintained at the set depth.
} 
many PIV systems, although the problem here (and for synthetic schlieren) is simplified somewhat by the fact that the individual pattern structures used in the matching procedure are conserved (provided the structures remain visible). This is not the case for PIV where particles can easily enter and leave the illuminated volume. A detailed description of the basic algorithm can be found in Dalziel et al. (2000), however a brief overview of the technique, and its more recent enhancements, is provided below.

Pattern matching algorithms typically find the displacement fields $\Delta x_{p}\left(x_{p}, y_{p}\right)$ and $\Delta y_{p}\left(x_{p}, y_{p}\right)$ that optimize some objective function. Here, $\left(x_{p}, y_{p}\right)$ are the image coordinates in (continuous) pixel space. In most PIV systems the goal is to maximize the cross correlation function, but, as shown by Dalziel et al. (2000), other measures of the difference may be used.

Let $P_{0}\left(x_{p}, y_{p}\right)$ and $P\left(x_{p}, y_{p}\right)$ denote the intensity in a typical reference and corresponding distorted image, respectively $\left(P_{0}\right.$ and $P$ will be piece-wise constant in $\left(x_{p}, y_{p}\right)$ across each pixel, with pixels centred on integer values of $x_{p}$ and $\left.y_{p}\right)$. For the present work we choose as our objective function the absolute difference between intensities in the two images,

$$
f\left(\delta x_{p}, \delta y_{p} ; x_{p}, y_{p}\right)=\left\langle\left|P\left(x_{p}+\delta x_{p}, y_{p}+\delta y_{p}\right)-P_{0}\left(x_{p}, y_{p}\right)\right|\right\rangle
$$

and seek to find the displacement field $\left(\Delta x_{p}, \Delta y_{p}\right)=\left(\delta x_{p}, \delta y_{p}\right)$ that minimizes this at the discrete pixel locations $\left(x_{i}, y_{j}\right)$ on a regular grid. Here $\langle\cdot\rangle$ denotes the average over all valid pixels ${ }^{3}$ located in some interrogation window $x_{i}-w_{x} \leqslant$ $x_{p} \leqslant x_{i}+w_{x}, y_{j}-w_{y} \leqslant y_{p} \leqslant y_{j}+w_{y}$. The displacement field that minimizes $f$ is determined with a multi-stage algorithm that yields subpixel accuracy and allows for distortions on scales much smaller than the size of the interrogation window.

Initially $f$ is optimized for integer values of $\left(\Delta x_{p}, \Delta y_{p}\right)$ to obtain a pixelresolution estimate for the optimal image displacements at the centre of the interrogation windows. A bi-quadratic least squares fit in the neighbourhood of this estimated minimum is then performed to provide the first level of sub-pixel refinement for this estimate. Spurious displacement vectors are detected at this point using a hierarchy of techniques, including the value of the objective function, curvature of the bi-quadratic fit, and median filtering. Spurious vectors are flagged and typically replaced by interpolation from neighbouring valid vectors.

In the second stage the algorithm is applied again, this time however the distorted image is interpolated (with either a bi-linear or bi-cubic kernel) so that the values of $P\left(x_{p}+\delta x_{p}, y_{p}+\delta y_{p}\right)$ used in $f$ have the noninteger subpixel displacements $\left(\delta x_{p}, \delta y_{p}\right)$ in the neighbourhood of the minimum $\left(\Delta x_{p}, \Delta y_{p}\right)$ identified by the first stage. Again, a bi-quadratic function is fitted in the neighbourhood of

\footnotetext{
${ }^{3}$ Some pixels may be excluded if, for example, a defect in the sensor or on the optical path means they do not form a valid part of the image of the pattern.
} 
the optimal displacements to improve this estimate further. This second stage typically resolves most or all of the spurious vectors identified on the first stage, although the validation procedure is repeated to identify any remaining spurious vectors prior to the third stage.

For the first two stages, the displacement field is calculated only at the centre of each of the interrogation windows, and this field applied uniformly across the entire interrogation window. In the third stage, however, the displacement field is extended via a bi-cubic interpolation to all pixels in the image and this extended displacement field is used in an attempt to remove the distortion from $P\left(x_{p}, y_{p}\right)$ by forming the distorted image

$$
Q\left(x_{p}, y_{p}\right)=P\left(x_{p}+\Delta \hat{x}_{p}, y_{p}+\Delta \hat{y}_{p}\right)
$$

where $\left(\Delta \hat{x}_{p}, \Delta \hat{y}_{p}\right)$ is the displacement field interpolated from $\left(\Delta x_{p}, \Delta x_{p}\right)$ at the discrete set of points $\left(x_{i}, y_{j}\right)$. The subpixel algorithm applied in the second stage is then applied to the image pair $P_{0}\left(x_{p}, y_{p}\right)$ and $Q\left(x_{p}, y_{p}\right)$ to obtain a correction to the displacement field $\left(\Delta x_{p}, \Delta y_{p}\right)$ for the centre of each interrogation region. This in turn may again be interpolated and the same algorithm re-applied, each time with a smaller subpixel increment in the neighbourhood of the current optimum.

The second and third stages described above could be applied iteratively, with successively smaller subpixel increments in the optimal search, to improve the estimate of the optimal pixel displacement, however the reduction in errors is slow and not warranted for the current problem due to the limitations in the pattern formed on the particle layer. Truncating this process after a small number of iterations leaves some zero-displacement noise in the estimated $\left(\Delta x_{p}, \Delta y_{p}\right)$ fields. This is a common but often overlooked artifact of subpixel pattern matching algorithms whereby taking $P\left(x_{p}, y_{p}\right)$ and $P_{0}\left(x_{p}, y_{p}\right)$ from the same image will not yield $\left(\Delta x_{p}, \Delta y_{p}\right)=0$, but will have a random noise that is an artifact of the patterns being matched. Here, the second stage iterates three times and the final stage once using $27 \times 27$ pixel interrogation windows at 17 pixel centres. The processing yielded a root mean square amplitude of around 0.005 pixels for the zero displacement noise. In our present case, this random noise is further reduced by the use of multiple different patterns, each with a different phase and a different seed to the random number generator.

For each of the nine experiments described in section 2, the set of reference images $P_{0}^{(l)}\left(x_{p}, y_{p}\right)$ and corresponding distorted images $P^{(l)}\left(x_{p}, y_{p}\right)$ (where $l$ denotes the pattern number) were processed using the pattern matching algorithm. This produced the corresponding displacement fields $\Delta x_{p}^{(l)}$ and $\Delta y_{p}^{(l)}$. The $27 \times 27$ pixel interrogation window used typically contained a pattern structure consisting of 4-6 dots (for the fine-scale structure patterns). The number of dots was limited primarily by an inability to form a sharp pattern with smaller features on the multiply-scattering particle bed. 
The optimal $\left(\Delta x_{p}, \Delta y_{p}\right)$ pattern displacement field is in pixel space $\left(x_{p}, y_{p}\right)$, whereas we need to know the bed displacements in physical space. In order to proceed, an accurate calibration to determine first the relationship between $\left(x_{p}, y_{p}\right)$ and the horizontal coordinates $(x, y)$ in the plane of the undisturbed sediment bed, and second the relationship between the pixel displacement field $\left(\Delta x_{p}, \Delta y_{p}\right)$ and the vertical bed deformation $\Delta z(x, y)$.

The system was calibrated using a rigid, horizontal plastic platform, $30 \mathrm{~cm} \times$ $30 \mathrm{~cm} \times 0.3 \mathrm{~cm}$, which could be adjusted to any reference height between $0.4 \mathrm{~cm}$ and $1.6 \mathrm{~cm}$. A coordinate grid was sketched on the reverse side of the platform, which was then adjusted to the zero reference height of $1 \mathrm{~cm}$, corresponding to the depth of the undisturbed sediment layer used in the experiments, and placed in the bottom of the tank. An image of the coordinate grid was captured, and used to establish the mapping from $\left(x_{p}, y_{p}\right)$ to $(x, y)$ in the zero plane.

The platform was then turned over, re-adjusted to the zero reference height and positioned back in the bottom of the tank. Each of the eleven patterns were projected onto the platform and an image captured. This procedure was repeated for a further ten reference heights from $z=0.5 \mathrm{~cm}$ to $z=1.5 \mathrm{~cm}$ (at intervals of $0.1 \mathrm{~cm}$ ), five above the $z=0$ plane and five below. For each corresponding pattern, the images at consecutive reference heights were processed using the pattern matching algorithm to calculate the displacement fields (at each discrete location $\left(x_{i}, y_{j}\right)$ ) produced by the incremental shifts of the platform height. The corresponding displacement fields obtained from the sequence of eleven patterns were then averaged and combined appropriately to produce

$$
\begin{aligned}
\Delta x_{i j k} & =\Delta x_{p}\left(x_{i}, y_{j}, \Delta z_{k}\right), \\
\Delta y_{i j k} & =\Delta y_{p}\left(x_{i}, y_{j}, \Delta z_{k}\right),
\end{aligned}
$$

i.e. the displacements produced at each location $\left(x_{i}, y_{j}\right)$ due to a vertical displacement $\Delta z_{k}=0.1 \times k \mathrm{~cm}$ about the zero reference height $z=0$.

Using the displacements $\Delta x_{i j k}$ and $\Delta y_{i j k}$ the corresponding (vertical) displacement function,

$$
\Delta z\left(x_{i}, y_{j}\right)=F\left(x_{i}, y_{j} ; \Delta x_{p}, \Delta y_{p}\right)
$$

could now be formulated. That is, for each location $\left(x_{i}, y_{j}\right)$ in the zero reference plane, $F$ returns the physical vertical displacement $\Delta z$ corresponding to the given pixel displacements $\Delta x_{p}$ and $\Delta y_{p}$. Initial analysis suggested, for the experimental setup described and for vertical displacements in the range $\pm 0.5 \mathrm{~cm}$, that both $\Delta x_{i j k}$ and $\Delta y_{i j k}$ were essentially invariant in $\left(x_{i}, y_{j}\right)$, and that $\Delta z\left(x_{i}, y_{j}\right)$ was dominated by the $y$-displacements, $\Delta y_{i j k}$. For example, $\Delta x_{i j 1} \approx 1$ pixel and $\Delta y_{i j 1} \approx 5$ pixels at each $\left(x_{i}, y_{j}\right)$, with standard deviations of order 0.1 pixel. Furthermore, for this range of displacements, $\Delta z$ had a clear linear dependence on $\Delta y_{i j k}$. 


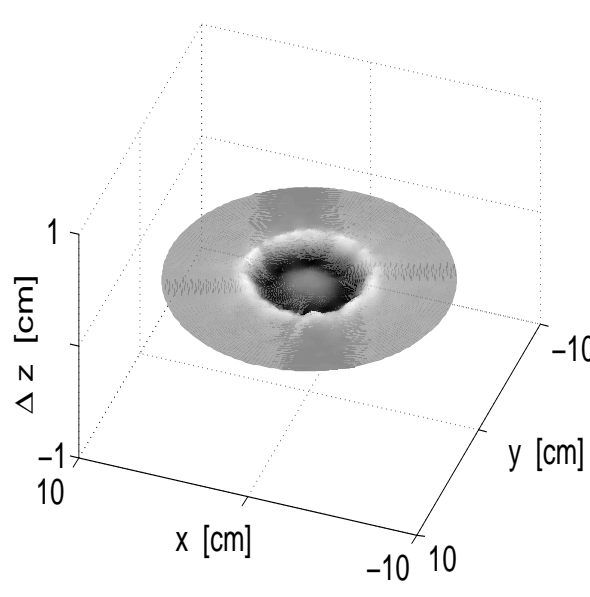

(a)

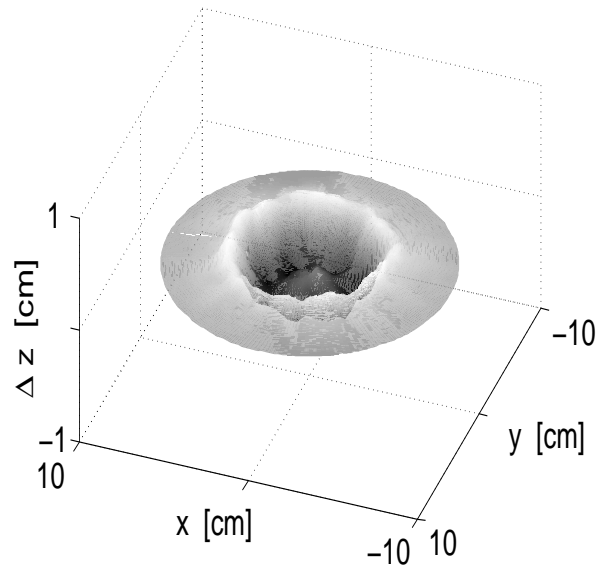

(b)

Figure 5: Corresponding to the two crater images shown in figure 4, the displacement fields $\Delta z(x, y)$ obtained using the pattern matching procedure, (a) $R e \approx 5000$, and (b) $R e \approx 28000$.

A comprehensive analysis, using several functional forms for $F$ was performed, confirming the above suggestions, i.e. that the vertical displacement field is adequately described by

$$
\Delta z\left(x_{i}, y_{j}\right)=F\left(\Delta y_{p}\right)=a \Delta y_{p}
$$

where for this particular calibration, $a=0.0217 \mathrm{~cm}$ per pixel. It is worth highlighting that the relative simplicity of (6) results from the fact that it is only valid for small scale vertical displacements in the range $|\Delta z| \leqslant 0.5 \mathrm{~cm}$. For larger scale vertical displacements we expect (6) to become invalid, and the nonlinear terms in $\left(\Delta x_{p}, \Delta y_{p}\right)$ to no longer be negligible. It is also worth noting that the experimental setup was designed in order to provide symmetry about the $y$-axis (in the plane of the layer) in an attempt to reduce the dependency on odd powers of $\Delta x_{p}$.

The displacement function (6) was applied to each of the $y$-displacement fields $\Delta y_{p}^{(l)}\left(x_{i}, y_{j}\right)$ obtained from the nine impact-crater images. For each crater, the resulting ensemble of $z$-displacement fields $\Delta z^{(l)}(x, y)$ were then averaged (over $l$ ). Two examples of the resulting (averaged) displacement fields $\Delta z(x, y)$, corresponding to the two crater images shown in figure 4, are given in figure 5 .

The displacement fields $\Delta z^{(l)}(x, y)$ were averaged to suppress noise introduced during the capturing of the images, and the subsequent processing using the pattern matching procedure ${ }^{4}$. An additional source of noise was produced

\footnotetext{
${ }^{4}$ Note that, as an initial attempt to reduce noise produced by the camera, each of the images $P_{0}^{(l)}\left(x_{p}, y_{p}\right)$ and $P^{(l)}\left(x_{p}, y_{p}\right)$ used in the processing procedure were obtained by time averaging sequential images of five frames (which corresponds to approximately $1.5 \mathrm{sec}$ ).
} 
when isolated regions of the pattern became hidden from the camera view due to the local curvature of the craters; typically these regions were located behind the front lip of the deeper crater (as viewed by the camera). This resulted in a large amplitude glitch in the corresponding region of the displacement field. These glitches were easily identified and removed using surrounding valid data to interpolate; this was done prior to the averaging.

As an indication of the amplitude of noise in the final (averaged) displacement fields, the standard deviation, $\sigma_{z}$, of $\Delta z(x, y)$ was calculated from isolated regions, away from the crater, where there was zero displacement. For each of the nine experiments, with maximum displacements ranging between 0.05$0.25 \mathrm{~cm}$, it was found that $0.001 \mathrm{~cm} \leqslant \sigma_{z} \leqslant 0.003 \mathrm{~cm}$. Using the maximum displacements as a measure of the signal strength, $S$, and $\sigma_{z}$ as a measure of the noise, the range of corresponding signal-to-noise ratios, $S / \sigma_{z}$, calculated from each of the experiments, was $50 \leqslant\left(S / \sigma_{z}\right) \leqslant 80$. Obtaining values for $\sigma_{z}$, again from regions corresponding to zero displacement, for each of the displacement fields $\Delta z^{(l)}(x, y)$ before the averaging, it was found that $0.002 \mathrm{~cm} \leqslant \sigma_{z} \leqslant 0.005 \mathrm{~cm}$, suggesting the noise was effectively halved by the averaging.

Comparing the crater images shown in figure 4 and the corresponding (measured) displacement fields in figure 5, there is a clear qualitative agreement between to two. Attempts were made, using a probe mounted on the top of the tank, to take direct measurements of the crater depths. However, the accuracy of the measurements obtained was unknown, and proved difficult to compare the displacement fields obtain using the pattern matching technique.

We note in passing that while the factor of two improvement by using multiple patterns is useful, it is less than might be anticipated. If each of the measurements had been statistically independent then we would have expected the noise to be reduced by a factor of $\sqrt{N}$ for $N$ patterns $($ i.e. $\approx 3.3$ ). The random patterns were designed to achieve close to independence by both phase shifting and using a different sequence of random numbers. Indeed, the maximum absolute normalized cross-correlation (for arbitrary shift) between captured images of any two patterns was 0.22 and the root mean square correlation was 0.02 , indicating we achieved close to this ideal (the peak correlation without shifting the images was around 0.02).

We believe the bulk of the difference between the observed error and that predicted from independence is due to thermal noise in the laboratory. As shown by Dalziel et al. (2000), synthetic schlieren is very sensitive to refractive index variations in the air, especially those occurring close to the camera lens. While conducting the experiments we believed that thermal effects would not significantly degrade the accuracy of the results. However, subsequent work on other experiments in the same part of the laboratory demonstrate that the 
signal due to thermal noise for the camera may have been in the region of 0.17 pixels. Scaling this for the present experiments, this predicts an error of up to $0.003 \mathrm{~cm}$, comparable with the error actually observed. Observations have shown that the time scale for these thermal fluctuations is comparable with the time scale for switching to the next pattern in the sequence, but short compared with the time elapsed between the images of the initial and final states being captured. Thus it is conceivable that the thermal effects remained correlated between the different patterns and would thus not be removed by the averaging process.

The projection equipment would also have been prone to thermal noise. However, we believe that this is less significant in the current setup due to the forced cooling of the data projector making the time scales much shorter and thus achieving closer to statistical independence.

\section{Validation}

To assess the accuracy of the results produced by the pattern matching technique described above, the procedure was applied to a deformed bedform with a known profile. Furthermore, to ascertain whether the technique could accurately measure the typical displacements and features found in the real craters (see figure 4), a profile was manufactured simulating these characteristics; namely, an axisymmetric profile with a radially varying depth.

The known crater was machined into a plastic disc of diameter $12 \mathrm{~cm}$ so that the depth at the outside edge was $1 \mathrm{~cm}$; the radial variation of the profile is shown in figure 6 (solid curve). The profile of the crater was measured using a dial test indicator (DTI), of the plunger type, located in the chuck head of a "turret" milling machine. With the aid of the milling machine's digital coordinate display, we were able to accurately traverse the DTI radially outwards from the centre of the crater ${ }^{5}$ at incremental steps of $0.1 \mathrm{~cm}$. The zero level of the DTI was set at the outside edge of the disc, and was able to measure displacements as small as $0.001 \mathrm{~cm}$. (Note that the radius of the profile and maximum displacement are approximately $5 \mathrm{~cm}$ and $0.3 \mathrm{~cm}$ respectively, which are similar to the features of the crater shown in figure $5(\mathrm{~b})$.)

A solid plastic plate, $30 \mathrm{~cm} \times 30 \mathrm{~cm} \times 1 \mathrm{~cm}$, was then prepared with a $12 \mathrm{~cm}$ diameter hole cut out of the centre to house the machined disc. Set at a height of $1 \mathrm{~cm}$, the adjustable calibration platform was placed at the bottom of the tank and used to obtain the sequence of reference images. The platform was replaced by the plate containing the known profile so that the corresponding sequence of deformed images could be captured. Using the procedure described

\footnotetext{
${ }^{5}$ The location of the crater centre was found by slowly moving the DTI over the central peak of the crater, from many different directions, to find the local maximum reading.
} 


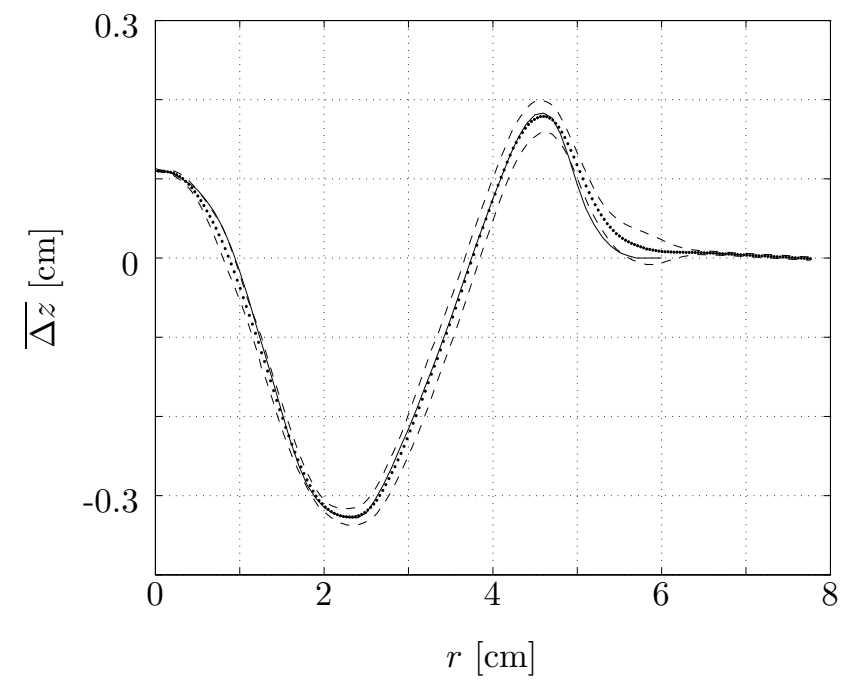

Figure 6: Plot showing results of the pattern matching technique applied to a known profile. The solid curve shows the radial variation of the known (axisymmetric) profile, the dotted curve shows the mean radial displacement profile, $\overline{\Delta z}$, obtained from the pattern matching technique, and the broken lines show $\overline{\Delta z} \pm \sigma_{z}$, where $\sigma_{z}$ is the standard deviation.

in section 3 , the set of reference and distorted images were processed to produce the displacement field $\Delta z(x, y)$.

To perform a detailed comparison between the known profile measurements and the displacement field $\Delta z(x, y)$, it was necessary to obtain the best possible alignment between the two. It was essential, therefore, to estimate, as accurately as possible, the position of the crater centre, $\left(x_{0}, y_{0}\right)$, in the displacement field $\Delta z(x, y)$. The following procedure was adopted to achieve this. Firstly, an initial estimate for the crater centre, $\left(\hat{x}_{0}, \hat{y}_{0}\right)$, was obtained from a contour plot of $\Delta z(x, y)$. Then, in the translated reference frame $\left(x^{\prime}, y^{\prime}\right)=\left(x-\hat{x}_{0}, y-\hat{y}_{0}\right)$, the known displacement field $\Delta z_{0}\left(x^{\prime}, y^{\prime}\right)$ was generated by interpolating the known profile measurements onto the regular grid $\left(x_{i}^{\prime}, y_{j}^{\prime}\right)$, i.e. the set of discrete locations in physical space corresponding to the centre of each image pixel. Using a similar method to the procedure described in section 3 , the objective function,

$$
\left\langle\left|\Delta z\left(x^{\prime}-\delta x^{\prime}, y^{\prime}-\delta y^{\prime}\right)-\Delta z_{0}\left(x^{\prime}, y^{\prime}\right)\right|\right\rangle,
$$

was then minimized to find optimal displacements $\left(\delta x^{\prime}, \delta y^{\prime}\right)=\left(\Delta x^{\prime}, \Delta y^{\prime}\right)$, effectively maximizing the correlation between the measured and known displacement fields. Hence, the final estimate for the location of the measured crater centre was given by $\left(x_{0}, y_{0}\right)=\left(\hat{x}_{0}+\Delta x^{\prime}, \hat{y}_{0}+\Delta y^{\prime}\right)$. Note that, in this case the averaging operation, $\langle\cdot\rangle$, was performed over the whole of the regular grid 


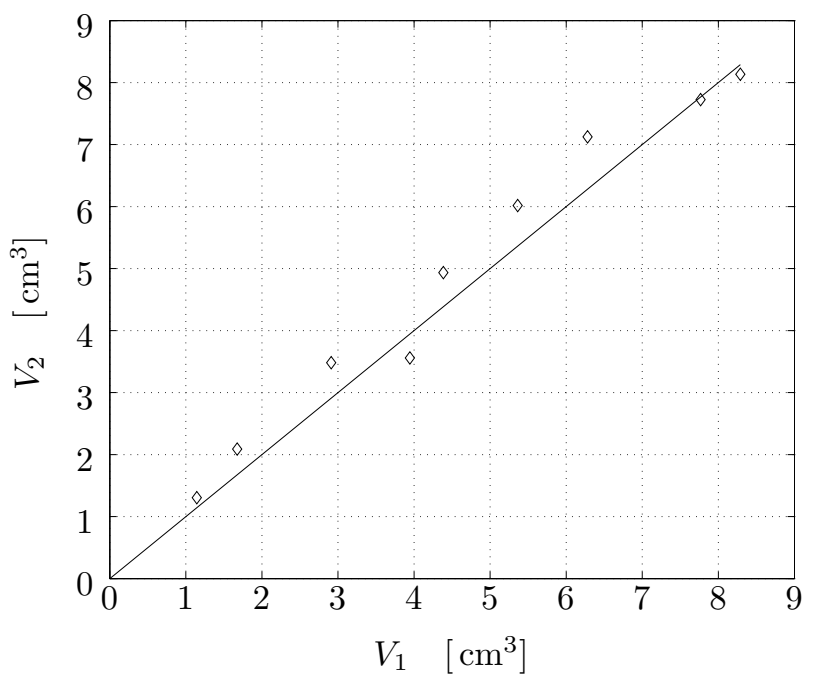

Figure 7: Plot comparing the volumes of deposited sediment, $V_{1}$, and eroded sediment, $V_{2}$, for each of the nine impact craters obtained from the experiments described in sections 2 and 3.

$\left(x_{i}^{\prime}, y_{j}^{\prime}\right)$, and a biquadratic fit was used to obtain sub-grid accuracy.

To provide a simple mechanism for comparing the results, the displacement field $\Delta z(x, y)$ was first transformed into polar coordinates,

$$
\begin{aligned}
& r=\sqrt{\left(x-x_{0}\right)^{2}+\left(y-y_{0}\right)^{2}}, \\
& \theta=\tan ^{-1}\left(\frac{y-y_{0}}{x-x_{0}}\right) .
\end{aligned}
$$

At each radial location the polar displacement field $\Delta z(r, \theta)$ was averaged over $\theta$ to produce the mean (radial) profile $\overline{\Delta z}(r)$. Figure 6 shows a comparison of the known profile (solid curve) with the mean profile $\overline{\Delta z}(r)$ (dotted curve) obtained from the pattern matching procedure. Also plotted, to give an indication of the error in the measurements, is the variation of one standard deviation, $\sigma_{z}(r)$, (broken curves) below and above the mean profile.

Clearly, the correspondence is excellent, with $\sigma_{z} \leqslant 0.03 \mathrm{~cm}$ at all locations along the profile. Also note that for $r>6 \mathrm{~cm}$, corresponding to the region where $\Delta z(r, \theta)=0, \sigma_{z}$ is constant and is of the same order as the corresponding values obtained from the experiments, i.e. $\sigma_{z}=0.003 \mathrm{~cm}$. Furthermore, a root mean square amplitude of $0.02 \mathrm{~cm}$ was returned from the crater region of $\Delta z(r, \theta)$, indicating that the error can be up to an order of magnitude greater in this region. This is due to the varying curvature of the crater distorting the shape of the actual pattern structures (i.e. dots) in the projected image, producing a 
greater error in the pattern matching procedure. However, for this test profile the signal-to-noise ratio $S / \sigma_{z}>10$ at all locations.

A secondary-level validation test was also applied to the displacement fields $\Delta z(x, y)$ obtained from the set of nine vortex ring experiments described in section 2. By mass conservation, the volume of eroded sediment must equal the volume of deposited sediment, and so

$$
\iint_{S} \Delta z(x, y) \mathrm{d} x \mathrm{~d} y=0
$$

where $S$ is the tank domain. The deposited sediment volume, $V_{1}$, and the eroded sediment volume, $V_{2}$, were defined respectively by

$$
\begin{aligned}
V_{1} & =\iint_{S} \Delta z^{+}(x, y) \mathrm{d} x \mathrm{~d} y, \\
V_{2} & =\iint_{S} \Delta z^{-}(x, y) \mathrm{d} x \mathrm{~d} y,
\end{aligned}
$$

where $\Delta z^{+}=\{\Delta z: \Delta z>0\}, \Delta z^{-}=\{\Delta z: \Delta z<0\}$. Figure 7 shows a comparison of $V_{1}$ plotted against $V_{2}$ for each of the nine experiments performed. The smallest and largest eroded volumes correspond to the impact craters produced by vortex rings with $R e \approx 5000$ and $R e \approx 28000$ respectively; the corresponding displacement fields are shown in figures 5 .

\section{Basic results}

The pattern matching procedure described in this paper was developed to provide a mechanism to analyse the fundamental characteristics of the impact crater produced by the interaction of a vortex ring with a sediment layer. This section provides a brief description of some of the basic, albeit fundamental crater characteristics, and illustrates how the measured displacement fields can be used to analyse them. This section is not, however, intended as a detailed description of the interaction itself. Hence, the dynamics of the interaction, and in particular the specific dynamics which cause the crater characteristics described below, have been omitted. These details can, however, be found in Munro \& Dalziel (2003).

One fundamental characteristic of the impact area is a radial deposit layer that forms at the outer edge of the crater, a typical example of which can be seen in figure 4(b). The characteristic shape of the known profile shown in figure 6 is typical of that of the mean profiles $\overline{\Delta z}(r)$ obtained from the measured craters, and the corresponding deposit layer is represented by the peak in profile between $r \approx 4 \mathrm{~cm}$ and $r \approx 6 \mathrm{~cm}$. Observations made during the experiments suggested that, over the range of $R e$ considered, the radial width of this deposition layer increased with Re. Hence, two important parameters that are used 


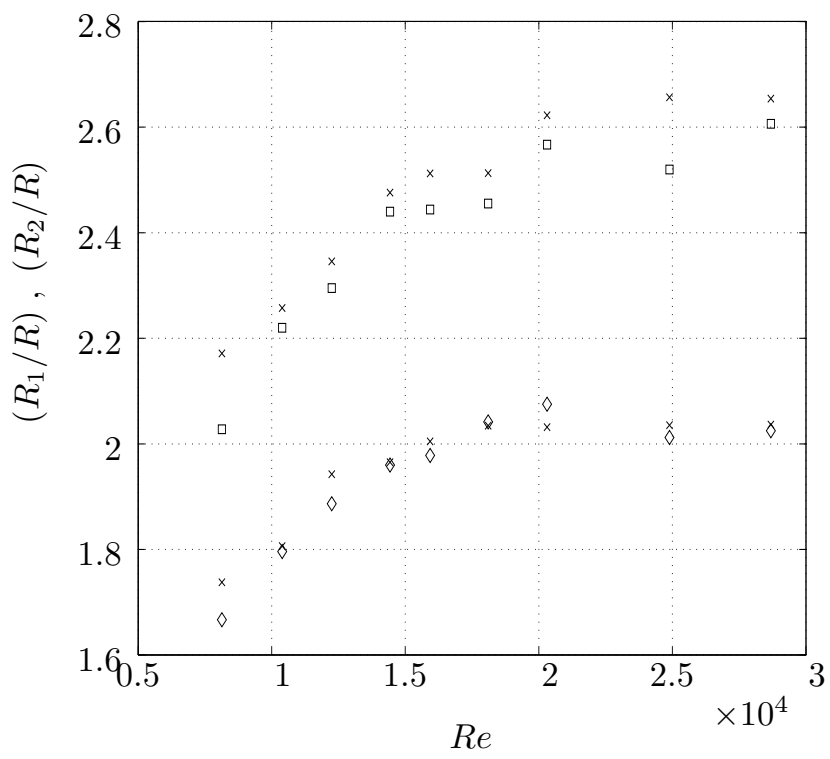

Figure 8: Estimates for the inner radius $R_{1}$ (diamonds) and the outer radius $R_{2}$ (squares), nondimensionalized by the outlet radius $R=1.95 \mathrm{~cm}$, obtained from the measured displacement fields $\Delta z(x, y)$, plotted against the corresponding vortex-ring Reynold's number Re. Also shown are the corresponding values obtained directly from the crater images (crosses).

characterize this feature are the internal (or crater) radius $R_{1}$, and the outer radius of the layer itself, $R_{2}$. Figure 8 shows the variation of $R_{1}$ (diamonds) and $R_{2}$ (squares), nondimensionalized by the outlet radius $R=D / 2=1.95 \mathrm{~cm}$, plotted against the corresponding vortex-ring Reynold's number, $R e$, for each of the nine experiments. The values of $R_{1}$ and $R_{2}$ shown are mean values obtained from the contour plot of the corresponding displacement field $\Delta z(x, y)$. In each case, the values were calculated from ten estimates of the corresponding diameters $2 R_{1}$ and $2 R_{2}$ obtained between the relevant $\Delta z=0$ contours. Also shown in figure 8 are estimates for $R_{1}$ and $R_{2}$ obtained directly from the crater images (crosses); two examples of the images used, at the upper and lower end of the $R e$ range, are shown in figure 4 . The overall qualitative features of the craters observed during the experiments are supported by these measurements, and show that both $R_{1}$ and $R_{2}$ initially increase monotonically with $R e$. However, beyond $R e \approx 2 \times 10^{4}$, both $R_{1}$ and $R_{2}$ appear to asymptote where, in particular, $R_{1} \approx 2 R$. We note, however, that in their study of resuspension due to wakes behind falling particles, Eames \& Dalziel (2000) saw that if sufficient material is resuspended, a particle-laden gravity current forms that leads to deposition over a much larger area. It is worth reiterating here that no resuspension was 


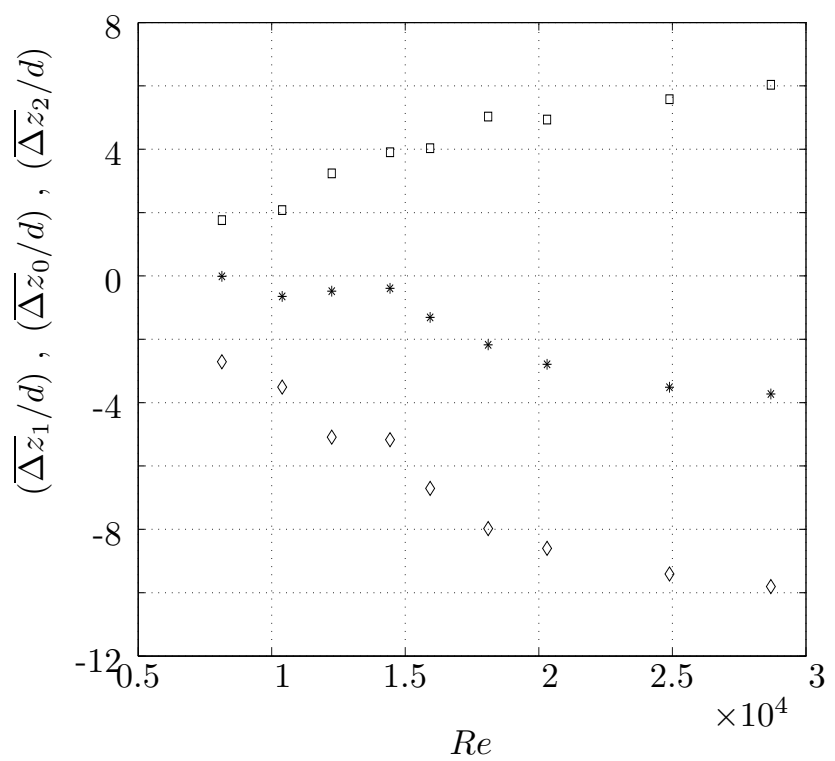

Figure 9: Plot showing the nondimensionalized crater-centre depth, $\overline{\Delta z}_{0} / d$ (stars), deposit layer height, $\overline{\Delta z}_{1} / d$ (squares), and crater depth, $\overline{\Delta z}_{2} / d$ (diamonds), against vortex-ring Reynolds number, Re. The values have been nondimensionalized using the characteristic particle diameter $d=0.025 \mathrm{~cm}$.

observed for $R e \lesssim 7.5 \times 10^{3}$.

Two other important features of the impact area to be considered here are the crater depth and the deposit layer height, to be denoted respectively by $\overline{\Delta z}_{1}$ and $\overline{\Delta z}_{2}$. As the notation suggests, the relevant measures used to characterize these two length scales have been obtained from the mean radial profile $\overline{\Delta z}(r)$, whereby $\overline{\Delta z}_{1}=\min (\overline{\Delta z})$ and $\overline{\Delta z}_{2}=\max (\overline{\Delta z})$. For example, for the known profile shown in figure 6 , the corresponding values are $\overline{\Delta z}_{1} \approx 0.18 \mathrm{~cm}$ and $\overline{\Delta z}_{2} \approx-0.32 \mathrm{~cm}$, occurring at $r \approx 2.3 \mathrm{~cm}$ and $r \approx 4.6 \mathrm{~cm}$ respectively. Figure 9 shows the values of $\overline{\Delta z}_{1}$ (squares) and $\overline{\Delta z}_{2}$ (diamonds), nondimensionalized by the characteristic particle diameter $d=0.025 \mathrm{~cm}$, against the vortex-ring Reynold's number, $R e$, for each of the nine experiments. As might be expected, the measurements show that $\overline{\Delta z}_{1}$ decreases, and $\overline{\Delta z}_{2}$ increases monotonically with $R e$, and again suggest there to be an eventual asymptotic state, albeit beyond some value of $R e$ outside the range considered here. Also shown in figure 9 is the crater-centre depth, $\overline{\Delta z}_{0}$ (stars), again, nondimensionalized by $d$.

\section{Discussion}


The technique outlined in this paper offers, for the first time, a non-intrusive method for obtaining accurate high-resolution measurements of sediment redeposition levels. In particular, the displacement field produced provides measurements at camera-resolution scales over the whole region of interest. The technique was originally designed to study redeposition craters produced by the interaction of a vortex ring with a sediment layer (Munro \& Dalziel, 2003). This specific application was used to illustrate the technique, and using a simulated crater with a known profile we were able to show that the technique is able to measure displacements comparable with an individual particle diameter.

One limitation of the technique is caused when large deformations of the sediment layer result in regions of pattern being hidden from the camera view. As a result, the patten matching algorithm is unable to identify the displacements in the corresponding regions. For the impact craters this typically occurs behind the front lip of the deeper craters. Attempts were made to combat this problem by using larger pattern structures (i.e. dots). An alternative, approach could be to modify the algorithm to utilize two cameras, deployed with orthogonal views (still both oblique to that of the projection). Then, pattern structures hidden from the view of one camera would typically still be in view of the second.

Consideration also needs to be given to the particles used. That is, the sediment layer needs to consist of material capable of sufficiently reflecting the projected patterns to produce a good contrasted image. For example, we found that for the glass ballotini particles with diameters above $0.06 \mathrm{~cm}$, the projected patterns were too diffuse. We predict that better results will be possible with smaller particles, and with opaque particles that provide a diffuse reflection rather than the complex optics of the present particles.

Although not documented here, the technique has also been applied over range of sediment layer depths (i.e. $0.2-1 \mathrm{~cm}$ ) and initial bedforms with sloping profiles. Preliminary analysis also suggests the technique can be applied to initial bedforms with a more complicated structure (e.g. saw tooth profiles). It should also be possible to apply the technique to analyse redeposition in sediment beds consisting of particles with a distribution of sizes.

\section{Acknowledgements}

The authors would like to thank Brian Dean, John Milton, David PageCroft, Trevor Parkin and Rob Raincock for technical assistance while assembling

the experimental equipment. Munro was provided with financial support by the 
EPSRC (grant number GR/R25620/01) and the Isaac Newton Trust.

\section{References}

Adrian RJ (1991) Particle-imaging techniques for experimental fluid mechanics. Ann Rev Fluid Mech 23: 261-304.

Cowen EA; Sveen JK (2004) Quantitative imaging techniques and their application to wavy flows. To appear in PIV and water waves, World Scientific, ed. J. Grue, P.L.F Liu \& G.K. Pedersen.

Dalziel SB; Hughes GO; Sutherland BR (2000) Whole field density measurements by 'synthetic schlieren'. Exp in Fluids 28: 322-335.

Eames I; Dalziel, SB (2000) Resuspension of dust by the flow around a sphere impacting a wall. J Fluid Mech 403: 305-328.

Glezer A (1988) The formation of vortex rings. Phys Fluids 31: 3532-3541.

Irish JL; White TE (1998) Coastal engineering applications of high resolution Lidar bathymetry. Coastal Eng 35: 47-71.

Linden PF; Turner JS (2001) The formation of 'optimal' vortex rings, and the efficiency of propulsion devices. J Fluid Mech 427: 61-72.

Munro RJ; Dalziel SB (2003) Particle resuspension by an impacting vortex ring. In Sediment and Sediment Transport, Kluwer Academic Publishers: 105-108.

Munro RJ; Dalziel SB (2004a) An electrical technique for measuring dynamic resuspension and subsequent redeposition levels. Submitted to Exp in Fluids.

Munro RJ; Dalziel SB (2004b) Attenuation technique for measuring sediment displacement levels. Submitted to Exp in Fluids.

Raffel M; Willert CE; Kompenhans (1998) Particle image velocimetry: a practical guide. Springer, Berlin.

Rooij F de; Dalziel SB; Linden PF (1999) Electrical measurement of sediment layer thickness under suspension flows. Exp in Fluids 26: 470-474.

Williams KL; Jackson DR (1998) Bistatic bottom scattering: model, experiments, and model/data comparison. J Acoustical Soc Am 103: 169-181. 